\title{
Icta 3iologica Gibirica
}

Journal of Biology

Founded in 2015

Altai State University

www.asu.ru

ISSN 2412-1908

Acta Biologica Sibirica, 2017, 3(1), 23-33

\section{A distribution list of the butterflies (Lepidoptera, Rhopalocera) of Tian-Shan within the boundaries of the former Soviet Union}

\author{
Stanislav K. Korb \\ Russian Entomological Society, Nizhny Novgorod Branch \\ P.O.Box 97 Nizhny Novgorod 603009 Russia.E-mail: stanislavkorb@list.ru
}

A distributive list of butterflies of Tian-Shan in borders of former Soviet Union is compiled, it contains 289 species: Hesperiidae - 21 species, Papilionidae - 21 species; Pieridae - 38 species, Satyridae - 67 species, Lybitheidae - 1 species, Danaidae - 1 species, Nymphalidae - 42 species, Riodinidae - 2 species, Lycaenidae - 96 species. New synonyms are established.

Key words: butterflies, fauna, Inner Tian-Shan, distributive list.

\section{Introduction}

The Tian-Shan mountains is a vast mountainous area located in Middle Asia and occupying countries: Uzbekistan, Kyrgyzstan, Kazakhstan, and China (not included to current study). Orographically this area divided into following parts: North Tian-Shan (including five mountain ridges: Kyrgyizsky, Transilian Alatau, Kungey Ala-Too, Terskey Ala-Too, Ketmen; and high-mountainous lake Issyk-Kul), West Tian-Shan (Talassky Mts. and located westwards mountain ridges as far as Syrdaryinsky Karatau Mts.), East Tian-Shan (Narat, Khalyktau and adjacent mountain ridges), Central Tian-Shan (SaryDzhas, Inylchektau, Kokshaaltau Mts.), Inner Tian-Shan (mountain ridges southwards Kyrghyz Mts. and Terskey Ala-Too Mts.) (Fig. 1).

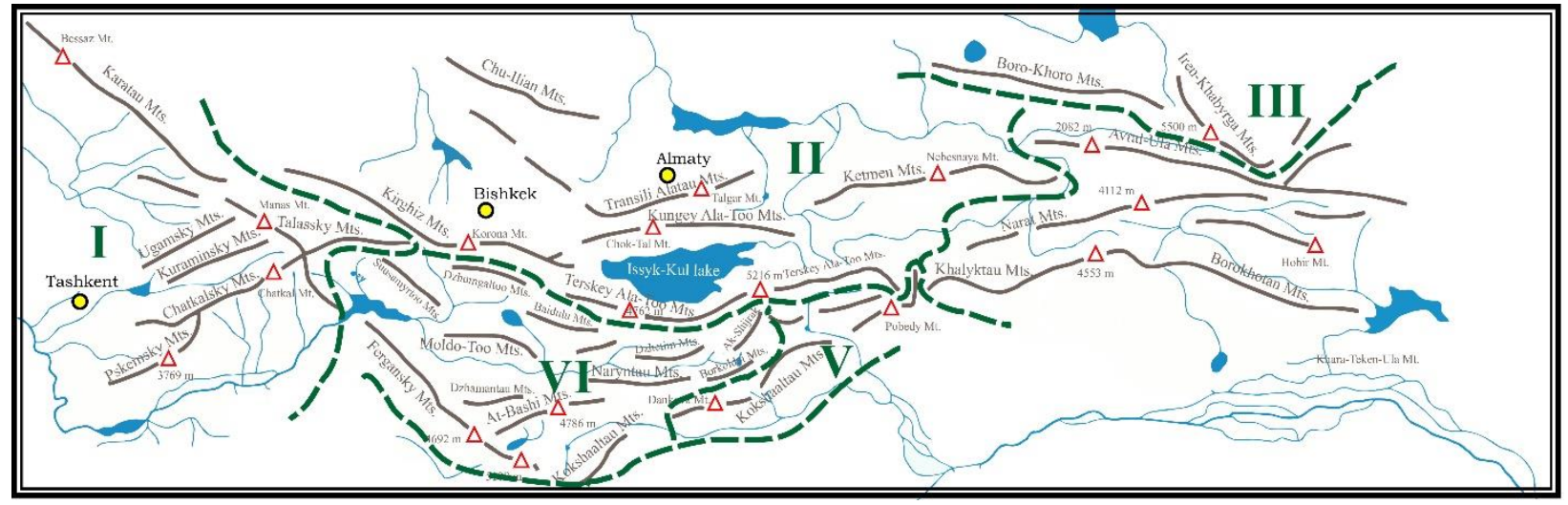

Fig. 1. Tian-Shan. I - West Tian-Shan, II - North Tian-Shan, III - Dzhungaria, IV - East Tian-Shan, V - Inner Tian-Shan, VI Central Tian-Shan. 
Butterfly fauna of this territory is rich and unique. The butterfly fauna of North Tian-Shan is studied better; the fauna of Central Tian-Shan studied not so good. In this paper, I compiled the list of butterflies of Tian-Shan.

\title{
Materials and Methods
}

Materials for this paper were:

1) Collections: Zoological Institute of the Russian Academy of Sciences (St.-Petersburg, Russia), Zoological Museum of the Moscow University (Moscow, Russia), Finnish Natural History Museum (Helsinki, Finland), Zoological Museum of the Nizhny Novgorod University (Nizhny Novgorod, Russia), Zoological Museum of Biological Institute of the Komi Science Centre of the Russian Academy of Sciences (Syktyvkar, Russia), Museum für Naturkunde an der Humboldt-Universität (Berlin, Germany), D.A. Pozhogin (Nizhny Novgorod, Russia), A.A. Shaposhnikov (Podolsk, Russia), tY.B. Kosarev (Nizhny Novgorod, Russia), L.V. Kaabak (Moscow, Russia), V.V. Titov (Zheleznodorozhny, Russia).

2) Author's own collections and researches in North, Inner and West Tian-Shan (1993-2016).

3) Published data.

\section{List of mountain ridges}

The following mountain ridges are included into parts of Tian-Shan (the Tian-Shan division after Gvozdetsky \& Mikhailov (1978); traditional Russian and/or Kyrgyzian names are given in square brackets):

Inner Tian-Shan: Akschijrak Mts. [Акшийрак, Ак-Шийрак]; Atbaschi Mts. [Атбаши, Ат-Баши]; Baibichetau Mts. [Байбичетоо, Байбичетау]; Baidula Mts. [Байдула]; Borkoldoi Mts. [Борколдой, Борколдай]; Dzhamantau Mts. [Джамантау, Жамантоо]; Dzhetim Mts. [Джетим, Жетим], Dzhetimbel Mts. [Джетимбель, Джетим-Бель, Жетим-Бель, Жөтим-Бел]; Dzhumgoltau Mts. [Джумголтау, Джумгалтоо, Жумгал-Too]; Fergansky Mts. [Ферганский хр.]; Moldo-Too Mts. [Молдо-Тоо, Мулдо-Тоо, Мулдатау]; Naryntau Mts. [Нарынтау, Нарынтоо]; Suusamyrtau Mts. [Cyусамыртау, Суусамыртоо, Сусамыртау].

Central Tian-Shan: Meridionalny Mts. [хр. Меридиональный]; Inylchektau Mts. [Иныльчектау, Эныльчектау, Эныльчек, Ингыльчек]; Kapkatas Mts. [Капкатас]; Karadzhorgo Mts. [Кара-Джорго, Kapa-Жорго]; Kekirimtau Mts. [Кекиримтау, Көкиримтоо]; Kokshaaltau Mts. [Кокшаалтау, Кокшаалтоо]; Sarydzhaz Mts. [Сары-Джаз, Сары-Жаз].

West Tian-Shan: Karamazor Mts. [Карамазор]; Karzhantau Mts. [Каржантау]; Kuraminsky Mts. [Кураминский хр.]; Pskem Mts. [Пскемский хр.]; Sandalashsky Mts. [Сандалашский хр.]; Syrdaryinsky Karatau Mts. [Сырдарьинский Кapaтay]; Talassky Mts. [Таласский хр.]; Chatkal Mts. [Чаткальский хр.]; Ugam Mts. [Угамский хр.].

North Tian-Shan: Ketmen Mts. [Кетмень]; Kirghiz Mts. [Киргизский хр., Ала-Too]; Terskey Ala-Too Mts. [Tepскей Ала-Тоо, Терскей Алатау]; Kungey Ala-Тoo Mts. [Кунгей Ала-Тоо. Кунгей Алатау]; Transili Alatau Mts. [Заилийский Алатау].

\section{PUBLISHED DATA}

Main papers on butterflies of Tian-Shan are: Alphéraky (1881), Korb (2010, 2011, 2012, 2015, 2017), Staudinger (1886, 1888, 1889), Tshikolovets (2003), Zhdanko (2005).

\section{LIST OF SPECIES}

\author{
Hesperiidae
}

Erynnis tages (Linnaeus, 1758). North Tian-Shan: Ketmen Mts.

E. marloyi (Boisduval, 1832). West Tian-Shan: Chatkalsky Mts.

Carcharodus alceae (Esper, [1780]). North Tian-Shan: all ridges; West Tian-Shan: all ridges; Inner Tian-Shan: Susamyrtoo, Dzhumgaltoo, Moldo-Too, Naryntoo, Ak-Shiyrak, Baidulu Mts.

Syrichtus antonia (Speyer, 1879). North Tian-Shan: all ridges; West Tian-Shan: Talassky, Fergansky, Chatkalsky Mts.; Inner Tian-Shan: Dzhumgaltoo, Suusamyrtoo, Naryn-Too, Moldo-Too, Baidulu Mts.

S. staudingeri (Speyer, 1879). North Tian-Shan: Kungey Ala-Too, Kirghizsky Mts.; Inner Tian-Shan: Naryntoo.

S. tessellum (Hübner, [1803]). North Tian-Shan: all ridges; West Tian-Shan: Talassky, Fergansky Mts.; Inner Tian-Shan: Dzhumgal-Too, Naryntoo, Moldo-Too, At-Bashi, Dzhetim-Bel, Baidulu Mts.; Central Tian-Shan: Sary-Dzhaz, Inylchektau Mts.

S. Iutulentus Grum-Grshimaïlo, 1887. North Tian-Shan: Kirghizsky, Transili Alatau Mts; West Tian-Shan: Syrdaryinsky Karatau, Talassky, Chatkalsky Mts.

Note. Taxon massageticus Zhdanko, 1993. Izv. NAN Kazakhstana., 2: 37 - 44 with type locality "Boraldai mountains" is a subspecies of lutulentus but not separate species.

S. nobilis (Staudinger, 1882). North Tian-Shan: Kirghizsky Mts.; Inner Tian-Shan: Suusamyrtoo, Moldo-Too Mts. 
S. proteus (Staudinger, 1886). North Tian-Shan: Kungey Ala-Too, Kirghizsky Mts.; West Tian-Shan: Talassky, Chatkalsky Mts.

Spialia geron (Watson, 1893). North Tian-Shan: Kirghizsky Mts.; West Tian-Shan: Chatkalsky Mts.

S. orbifer (Hübner, [1823]). North Tian-Shan: all ridges; West Tian-Shan: Syrdaryinsky Karatau, Talassky, Chatkalsky, Ugamsky, Fergansky Mts.; Inner Tian-Shan: Suusamyrtoo, Dzhumgaltoo, Naryntoo, Moldo-Too, Baidulu Mts.

Pyrgus sidae (Esper, [1782]). North Tian-Shan: Kirghizsky, Kungey Ala-Too Mts.; West Tian-Shan: Syrdaryinsky Karatau, Chatkalsky Mts.; Inner Tian-Shan: Dzhumgaltoo, Suusamyrtoo, Naryntoo Mts.

P. malvae (Linnaeus, 1758). North Tian-Shan: all ridges; West Tian-Shan: Talassky, Fergansky Mts.; Inner Tian-Shan: Moldo-Too Mts.

P. alpinus (Erschoff, 1874). North Tian-Shan: all ridges; West Tian-Shan: all ridges; Inner Tian-Shan: Dzhumgaltoo, Suusamyrtoo, Baidulu, Moldo-Too, Naryntoo, Dzhetim-Bel, Borkoldoi, At-Bashi Mts; Central Tian-Shan: Inylchek, SaryDzhaz, Kokshaaltoo Mts.

Thymelicus lineola (Ochsenheimer, [1808]). North Tian-Shan: all ridges; West Tian-Shan: Syrdaryinsky Karatau, Talassky, Chatkalsky, Fergansky Mts.; Inner Tian-Shan: Suusamyrtoo, Dzhumgaltoo, Baidulu, Naryntoo, Dzhetim-Bel, Borkoldoi, Moldo-Too Mts.

T. alaicus (Filipjev, 1931). West Tian-Shan: Fergansky, Chatkalsky Mts.; Inner Tian-Shan: Suusamyrtoo, Dzhumgaltoo Mts.

Hesperia comma (Linnaeus, 1758). All ridges.

H. sy/vanus (Esper, [1777]). North Tian-Shan: Kirghizsky Mts.; West Tian-Shan: Talassky, Chatkalsky, Fergansky Mts.; Inner Tian-Shan: Naryntoo, Moldo-Too, Baidulu Mts.

H. thibetana Oberthür, 1886. Inner Tian-Shan: At-Bashi, Naryntoo Mts.

Eogenes alcides (Herich-Schäffer, [1852]). West Tian-Shan: Fergansky Mts.

Gegenes nostrodamus (Fabricius, 1793). West Tian-Shan: Fergansky Mts.

$$
\text { Papilionidae }
$$

Papilio alexanor Esper, [1799]. North Tian-Shan: Kirghizsky Mts.; West Tian-Shan: Chatkalsky, Fergansky Mts.

P. machaon Linnaeus, 1758. All ridges.

Iphiclides podalirius (Linnaeus, 1758). North Tian-Shan: Kirghizsky, Ketmen, Transili Alatau-Mts.

Hypermnestra helios (Nickerl, 1846). North Tian-Shan: Kirghizsky, Kungey Ala-Too, Transili Alatau Mts.; West TianShan: Syrdaryinsky Karatau, Chatkalsky Mts.

Parnassius apollonius (Eversmann, 1847). North Tian-Shan: Kirghizsky, Kungey Ala-Too, Ketmen, Transili Alatau Mts.; West Tian-Shan: Syrdaryinsky Karatau, Karzhantau, Pskemsky, Ugamsky, Chatkalsky, Talassky, Fergansky Mts.; Inner TianShan: Dzhumgaltoo, Suusamyrtoo, Moldo-Too, Naryntoo, Dzhetim-Bel Mts.

P. actius (Eversmann, 1843). All ridges.

P. jacquemonti Boisduval, 1836. West Tian-Shan: Fergansky Mts.

P. tianschanicus Oberthür, 1879. All ridges.

P. apollo (Linnaeus, 1758). North Tian-Shan: all ridges; West Tian-Shan: Talassky.; Inner Tian-Shan: Dzhumgaltoo Mts.

Driopa mnemosyne (Linnaeus, 1758). North Tian-Shan: all ridges; West Tian-Shan: all ridges; Inner Tian-Shan: Borkoldoi, At-Bashi, Dzhetim-Bel Mts.

Kreizbergius boedromius (Püngeler, 1901). North Tian-Shan: Kirghizsky, Transili Alatau, Terskey Ala-Too, Kungey Ala-

Too Mts.; West Tian-Shan: Talassky Mts.; Inner Tian-Shan: Suusamyrtoo, Dzhetim-Bel, At-Bashi, Borkoldoi Mts.; Central

Tian-Shan: Inylchektau, Sary-Dzhaz, Kokshaaltoo Mts.

K. simo (Gray, 1853). Central Tian-Shan: Kokshaaltoo Mts.

K. simonius (Staudinger, 1889). Inner Tian-Shan: Dzhamantau Mts.

Koramius patricius (Niepelt, 1911). North Tian-Shan: Kirghizsky, Kungey Ala-Too, Terskey Ala-Too, Transili Alatau Mts.;

West Tian-Shan: Chatkalsky, Talassky Mts.; Inner Tian-Shan: Suusamyrtoo, Baidulu, At-Bashi, Borkoldai, Dzhetim-Bel Mts.;

Central Tian-Shan: Inylchektau, Sary-Dzhaz, Kokshaaltoo Mts.

K. priamus (Bryk, 1914). North Tian-Shan: Terskey Ala-Too Mts.; Central Tian-Shan: sarydzhaz, Inylchektau, Kaindy, Meridionalny, Kokshaaltoo Mts.; Inner Tian-Shan: Borkoldai Mts.

K. delphius (Eversmann, 1843). North Tian-Shan: all ridges; West Tian-Shan: all ridges; Inner Tian-Shan: Suusamyrtoo,

Dzhumgaltoo, Moldo-Too, Baidulu, Naryntoo, At-Bashi, Dzhetim-Bel Mts.; Central Tian-Shan: all ridges.

K. maximinus (Staudinger, 1891). West Tian-Shan: Karzhantau, Pskemsky, Ugamsky, Sandalashsky, Koksuysky,

Chatkalsky, Talassky, Kuraminski Mts.

K. staudingeri (A.Bang-Haas, 1882). Inner Tian-Shan: Dzhamantau, Baubash-Ata Mts.

K. Charltonius (Gray, 1853). Inner Tian-Shan: Dzhamantau Mts.

K. davydovi (Churkin, 2006). Inner Tian-Shan: Moldo-Too Mts.

K. Ioxias (Püngeler, 1901). Central Tian-Shan: Kaindy, Inylchektau, Kokshaaltoo Mts. 
Pieridae

Leptidea juvernica Williams, 1946. North Tian-Shan: Terskey Ala-Too Mts.

L. descimoni Mazel, 2004. North Tian-Shan: Terskey Ala-Too Mts.

L. sinapis (Linnaeus, 1758). North Tian-Shan: all ridges; West Tian-Shan: Talassky, Chatkalsky, Fergansky Mts.

Colias cocandica Erschoff, 1874. North Tian-Shan: all ridges; West Tian-Shan: Talassky, Chatkalsky, Ugamsky, Pskemsky, Fergansky Mts.; Inner Tian-Shan: Baidulu, At-Bashi, Naryntau, Dzhetim-Bel Mts.; Central Tian-Shan: all ridges.

C. alta Staudinger, 1886 (=C. worthyi Zhdanko, [2012]). North Tian-Shan: Kirghizsky Mts.; Inner Tian-Shan: Naryntoo, At-Bashi Mts, Baidulu Mts.

C. grieshuberi Korb, 2004. North Tian-Shan: all ridges; West Tian-Shan: Chatkalsky Mts.

C. erate (Esper, [1801]). All ridges.

C. ionovi Korb, 2005. North Tian-Shan: Kirghizsky, Transili Alatau Mts.

C. romanovi Grum-Grshimailo, 1885. North Tian-Shan: all ridges; West Tian-Shan: Talassky, Chatkalsky, Ugamsky, Fergansky Mts.; Inner Tian-Shan: Ak-Shiyrak, Moldo-Too, Baidulu, Naryntoo, At-Bashi Mts.

C. staudingeri Alphéraky, 1881. North Tian-Shan: all ridges; Inner Tian-Shan: all ridges; Central Tian-Shan: all ridges.

C. regia Grum-Grshimailo, 1887. Inner Tian-Shan: Baidulu, Nur, Naryntoo Mts.; Central Tian-Shan: all ridges.

C. thisoa Ménétriès, 1832. North Tian-Shan: all ridges; West Tian-Shan: Chatkalsky, Fergansky, Talassky Mts.; Inner

Tian-Shan: Moldo-Too, Baidulu, Nur, Suusamyrtoo, Naryntau, At-Bashi Mts.

C. erschoffi Alphéraky, 1881. North Tian-Shan: Ketmen Mts.

C. draconis Grum-Grshimailo, 1891. West Tian-Shan: Talassky, Chatkalsky, Pskemsky, Kuraminsky Mts.

Gonepteryx rhamni (Linnaeus, 1758). North Tian-Shan: all ridges.

G. farinosa Zeller, 1847. North Tian-Shan: Kirghizsky Mts.; West Tian-Shan: Chatkalsky, Fergansky, Talassky, Kuraminsky, Pskemsky Mts.

Anthocharis cardamines (Linnaeus, 1758). North Tian-Shan: all ridges; West Tian-Shan: all ridges.

Euchloe ausonia (Hübner, [1803]). North Tian-Shan: Kirghizsky, Kungey Ala-Too, Ketmen, Transili Alatau Mts.

E. daphalis (Moore, 1865). North Tian-Shan: Kirghizsky Mts.; West Tian-Shan: Talassky, Chatkalsky, Fergansky Mts.; Inner Tian-Shan: Baidulu, Naryntoo, Suusamyrtoo, Dzhumgaltoo Mts.

Zegris eupheme (Esper, [1805]). North Tian-Shan: Kirghizsky Mts.

Z. fausti Christoph, 1877. North Tian-Shan: Transili Alatau, Ketmen Mts.; West Tian-Shan: Ugamsky, Kuraminsky, Karzhantau Mts.

Microzegris pyrothoe (Eversmann, 1832). North Tian-Shan: Transili Alatau Mts.

Aporia crataegi (Linnaeus, 1758). All ridges.

Metaporia leucodice (Eversmann, 1843). All ridges.

Pieris brassicae (Linnaeus, 1758). North Tian-Shan: all ridges; West Tian-Shan: all ridges; Inner Tian-Shan: Baidulu, Naryntoo, Dzhetim-Bel Mts.

P. deota (de Nicéville, [1884]). North Tian-Shan: Kirghizsky, Terskey Ala-Too Mts.; Inner Tian-Shan: Dzhetim-Bel, Naryntoo Mts.; Central Tian-Shan: Kokshaaltoo Mts.

P. napi (Linnaeus, 1758). All ridges.

P. euorientis Verity, [1908]. North Tian-Shan: Kirghizsky Mts.

P. banghaasi Sheljuzhko, 1910. North Tian-Shan: Kirghizsky, Kungey Ala-Too Mts.; Inner Tian-Shan: Kara-Dzhorgo, Naryntoo, Nura, Baidulu Mts.

P. rapae (Linnaeus, 1758). All ridges.

P. ochsenheimeri Staudinger, 1886. North Tian-Shan: Kirghizsky Mts.; West Tian-Shan: Talassky, Chatkalsky, Fergansky Mts.; Inner Tian-Shan: Suusamurtoo, Dzhumgaltoo Mts.

P. canidia (Sparrman, 1768). North Tian-Shan: Kirghizsky, Transili Alatau Mts.; West Tian-Shan: all ridges; Inner TianShan: Dzhumgaltoo, Suusamyrtoo Mts.

P. krueperiStaudinger, 1860. North Tian-Shan: Kirghizsky Mts.; West Tian-Shan: Chatkalsky, Talassky, Fergansky Mts.

Pontia chloridice (Hübner, [1813]). North Tian-Shan: all ridges; West Tian-Shan: Syrdaryinsky Karatau Mts.; Inner Tian-Shan: Baidulu, Naryntoo Mts.

P. daplidice (Linnaeus, 1758). All ridges.

P. callidice (Hübner, [1800]). All ridges.

Baltia shawii (Bates, 1873). Inner Tian-Shan: Moldo-Too Mts.

Lybitheidae

Lybithea celtis (Laicharting, 1782). North Tian-Shan: Kirghizsky, Kungey Ala-Too, Transili Alatau Mts.; West Tian-Shan: all ridges. 
Danaidae

Danaus chrysippus (Linnaeus, 1758). North Tian-Shan: Kirghizsky Mts.; West Tian-Shan: Chatkalsky, Karzhantau, Pskemsky Mts.

$$
\text { Nymphalidae }
$$

Limenitis lepechini Erschoff, 1874. West Tian-Shan: Fergansky Mts.

Neptis rivularis (Scopoli, 1763). North Tian-Shan: all ridges; West Tian-Shan: Chatkalsky, Talassky, Fergansky Mts.; Inner Tian-Shan: Suusamyrtoo, Dzhumgaltoo Mts.

Argynnis pandora ([Denis et Schiffermüller], 1775). North Tian-Shan: all ridges; West Tian-Shan: all ridges; Inner TianShan: all ridges; Central Tian-Shan: Inylchektau, Sary-Dzhaz Mts.

A. paphia (Linnaeus, 1758). North Tian-Shan: all ridges; West Tian-Shan: Chatkalsky, Talassky, Fergansky Mts.

A. niobe (Linnaeus, 1758). All ridges.

A. adippe (Linnaeus, 1767). North Tian-Shan: all ridges; West Tian-Shan: Chatkalsky, Talassky, Fergansky Mts.

A. aglaja (Linnaeus, 1758). All ridges.

Issoria lathonia (Linnaeus, 1758). All ridges.

Brenthis ino (Rottemburg, 1775). North Tian-Shan: all ridges; West Tian-Shan: Talassky, Chatkalsky Mts.; Inner TianShan: Suusamyrtoo, Dzhumgaltoo Mts.

B. hecate ([Denis et Schiffermüller], 1775). North Tian-Shan: Kirghizsky, Kungey Ala-Too, Transili Alatau Mts.; West Tian-Shan: Talassky, Chatkalsky, Ugamsky, Fergansky Mts.; Inner Tian-Shan: all ridges.

Boloria erubescens (Staudinger, 1901). All ridges.

B. generator (Staudinger, 1886). All ridges.

B. dia (Linnaeus, 1764). North Tian-Shan: Kirghizsky Mts.

Polygonia c-album (Linnaeus, 1758). North Tian-Shan: all ridges; West Tian-Shan: Chatkalsky, Talassky, Fergansky Mts.; Inner Tian-Shan: Naryntoo, Baidulu, Karakatty, Dzhumgoltau Mts.

P. egea (Cramer, [1775]). North Tian-Shan: all ridges; West Tian-Shan: all ridges; Central Tian-Shan: Sary-Dzhaz Mts.

Nymphalis vau-album ([Denis et Schiffermüller], 1775). North Tian-Shan: all ridges; West Tian-Shan: Chatkalsky, Talassky, fergansky Mts.; Inner Tian-Shan: Moldo-Too, Naryntoo Mts.

N. xanthomelas ([Denis et Schiffermüller], 1775). North Tian-Shan: all ridges; West Tian-Shan: all ridges; Inner TianShan: At-Bashi, Naryntoo, Baidulu Mts.

N. antiopa (Linnaeus, 1758). North Tian-Shan: all ridges; West Tian-Shan: all ridges; Inner Tian-Shan: Naryntoo, Baidulu Mts.

Aglais urticae (Linnaeus, 1758). All ridges.

Inachis io (Linnaeus, 1758). North Tian-Shan: all ridges; West Tian-Shan: Talassky, Chatkalsky Mts.; Inner Tian-Shan: Suusamyrtoo, Dzhumgaltoo Mts.

Vanessa atalanta (Linnaeus, 1758). North Tian-Shan: Kirghizsky, Transili Alatau Mts.; West Tian-Shan: Chatkalsky, Talassky Mts.

V. cardui (Linnaeus, 1758). All ridges.

Euphydryas alexandrina (Staudinger, 1887). North Tian-Shan: all ridges; Inner Tian-Shan: Kara-Dzhorgo, Baidulu, Dzhetim-Bel, Dzhumgaltoo, Naryntoo, At-Bashi; Central Tian-Shan: Sary-Dzhaz, Inylchektau Mts.

Melitaea didyma (Esper, [1779]). North Tian-Shan: all ridges; West Tian-Shan: all ridges; Inner Tian-Shan: Dzhetim-Bel, Moldo-Too, Baidulu Mts.

M. persea Kollar, [1849]. North Tian-Shan: Kungey Ala-Too, Terskey Ala-Too, Kirghizsky Mts.; West Tian-Shan: Talassky Mts.

M. ala Staudinger, 1881 (=M. kotshubeji Sheljuzhko, 1929). North Tian-Shan: all ridges; West Tian-Shan: Chatkalsky

Mts., Fergansky Mts., Talassky Mts.; Inner Tian-Shan: all ridges; Central Tian-Shan: Kokshaaltoo Mts.

M. ninae Sheljuzhko, 1935. North Tian-Shan: Kirghizsky Mts.; West Tian-Shan: Talassky, Chatkalsky Mts.

M. enarea Fruhstorfer, 1916. West Tian-Shan: Fergansky Mts.

M. Iunulata Staudinger, 1901. North Tian-Shan: Kirghizsky, Kungey Ala-Too, Terskey Ala-Too, Transili Alatau Mts.; West Tian-Shan: all ridges.

M. fergana Staudinger, 1882. North Tian-Shan: all ridges; West Tian-Shan: Talassky Mts.; Inner Tian-Shan: all ridges; Central Tian-Shan: Inylchektau, Sary-Dzhaz Mts.

M. athene Staudinger, 1881. North Tian-Shan: Ketmen, Transili Alatau Mts.

M. robertsi Butler, 1880. West Tian-Shan: all ridges; Inner Tian-Shan: Naryntoo, Moldo-Too Mts.

M. minerva Staudinger, 1881. North Tian-Shan: Kirghizsky, Ketmen, Kungey Ala-Too, Transili Alatau Mts.; West TianShan: all ridges; Inner Tian-Shan: Songkeltoo, Moldo-Too, Dzhetim-Bel, Baidulu Mts.

M. pallas Staudinger, 1886. North Tian-Shan: Kirghizsky Mts.; West Tian-Shan: Talassky, Chatkalsky Mts.; Inner TianShan: all ridges.

M. asteroida Staudinger, 1881. North Tian-Shan: all ridges; West Tian-Shan: Talassky Mts.; Inner Tian-Shan: all ridges. 
M. palamedes Grum-Grshimaïlo, 1890. North Tian-Shan: Transili Alatau; West Tian-Shan: Talassky Mts.; Inner TianShan: Dzhumgaltoo Mts.

M. turanica Erschoff, 1874. West Tian-Shan: Chatkalsky Mts.

M. ornata Christoph, 1893. North Tian-Shan: Kirghizsky, Kungey Ala-Too, Transili Alatau Mts.; West Tian-Shan: Talassky Mts.

M. sibina Alphéraky, 1881. North Tian-Shan: Kirghizsky, Ketmen, Transili Alatau, Kungey Ala-Too Mts.; West TianShan: all ridges; Inner Tian-Shan: Suusamyrtoo, Dzhumgaltoo, Moldo-Too, Naryntoo, Baidulu Mts.

M. arduinna (Esper, [1784]). North Tian-Shan: Kirghizsky, Transili Alatau Mts.; West Tian-Shan: all ridges; Inner TianShan: Dzhumgaltoo, Moldo-Too, Naryntoo, Baidulu Mts.

M. cinxia (Linnaeus, 1758). North Tian-Shan: Kirghizsky, Kungey Ala-Too Mts.; West Tian-Shan: Chatkalsky, Talassky, Fergansky Mts.; Inner Tian-Shan: Suusamyrtoo, Dzhumgaltoo, Moldo-Too, Naryntoo Mts.

\section{Satyridae}

Lasiommata maera (Linnaeus, 1758). North Tian-Shan: Ketmen, Kungey Ala-Too Mts.

L. menava Moore, 1865. North Tian-Shan: Kirghizsky Mts.; West Tian-Shan: all ridges. Marginarge eversmanni (Eversmann, 1847). All ridges, except Central Tian-Shan.

Melanargia russiae (Esper, [1786]). North Tian-Shan: Kirghizsky, Kungey Ala-Too, Transili Alatau, Ketmen Mts.

M. parce Staudinger, 1882. All ridges, except Central Tian-Shan.

Triphysa issykkulica Dubatolov, Korb et Yakovlev, 2016. North Tian-Shan: Kirghizsky, Kungey Ala-Too, Transili Alatau Mts.

Lyela myops (Staudinger, 1881). North Tian-Shan: all ridges; West Tian-Shan: Chatkalsky, Ugamsky, Pskemsky, Syrdaryinsky Karatau, Karzhantau Mts.

Disommata nolckeni (Erschoff, 1874). North Tian-Shan: Kirghizsky Mts.; West Tian-Shan: all ridges; Inner Tian-Shan: At-Bashi, Naryntoo, Moldo-Too, Dzhetim-Bel Mts.

Chortobius tullia (Müller, 1764). North Tian-Shan: Kungey Ala-Too, Kirghizsky Mts.; West Tian-Shan: all ridges; Inner Tian-Shan: Dzhumgaltoo Mts.

C. mahometana (Alphéraky, 1881). North Tian-Shan: all ridges; Inner Tian-Shan: baidulu Mts.

C. sunbecca (Eversmann, 1843). All ridges.

C. pamphilus (Linnaeus, 1758). North Tian-Shan: all ridges; West Tian-Shan: all ridges; Inner Tian-Shan: Dzhumgaltoo, Suusamyrtoo Mts.

Paralasa helios (O.Bang-Haas, 1927). Central Tian-Shan: all ridges; Inner Tian-Shan: Naryntoo Mts.

P. bogutena V.Lukhtanov et A.Lukhtanov, 1994. North Tian-Shan: Kirghizsky, Kungey Ala-Too, Terskey Ala-Too, Transili Alatau Mts.

P. kusnezovi (Avinov, 1910). North Tian-Shan: Kirghizsky Mts.; West Tian-Shan: all ridges; Inner Tian-Shan: Suusamyrtoo, Moldo-Too, Dzhumgaltoo Mts.

Proterebia afra (Fabricius, 1787). North Tian-Shan: Kirghizsky, Transili Alatau Mts.; West Tian-Shan: Syrdaryinsky Karatau Mts.

Erebia turanica Erschoff, 1877. North Tian-Shan: all ridges; West Tian-Shan: Chatkalsky, Talassky Mts.; Inner TianShan: Suusamyrtoo, Dzhumgaltoo, Moldo-Too Mts.; Central Tian-Shan: Kaindy, Inylchektau Mts.

E. meta Staudinger, 1886. West Tian-Shan: Chatkal, Fergansky Mts.; Inner Tian-Shan: Suusamyrtoo, Dzhumgaltoo Mts.

E. mopsos Staudinger, 1886. North Tian-Shan: all ridges; West Tian-Shan: Chatkalsky, Talassky Mts.; Inner Tian-Shan: all ridges; Central Tian-Shan: all ridges.

E. mongolica Erschoff, 1888. North Tian-Shan: all ridges; West Tian-Shan: Talassky, Chatkalsky Mts.; Inner Tian-Shan: all ridges; Central Tian-Shan: Sary-Dzhaz Mts.

E. saita Korb, 2010. Inner Tian-Shan: Dzhetim-Bel Mts.

E. radians Lang, 1884. North Tian-Shan: all ridges; West Tian-Shan: Fergansky Mts.; Inner Tian-Shan: Dzhumgaltoo, Suusamyrtoo, At-Bashi Mts.

E. sokolovi Lukhtanov, 1990. North Tian-Shan: Kirghizsky Mts.; West Tian-Shan; Talassky, Chatkalsky Mts.

E. kalmuka Alphéraky, 1881. North Tian-Shan: Terskey Ala-Too Mts.; Inner Tian-Shan: Baidulu, Naryntoo, Moldo-Too, Dzhetim-Bel, Borkoldai, At-Bashi; Central Tian-Shan: all ridges.

E. eugenia Churkin, 2000. Central Tian-Shan: Kokshaaltoo Mts.

Karanasa talastauana (O.Bang-Haas, 1927). West Tian-Shan: all ridges.

K. kirgizorum Avinoff et Sweadner, 1951. North Tian-Shan: Kirghizsky Mts.

K. wilkinsi (Erschoff, 1881). North Tian-Shan: Transili Alatau, Kungey Ala-Too Mts.; West Tian-Shan: Talassky, Fergansky Mts.; Inner Tian-Shan: At-Bashi, Moldo-Too, Baidulu, Songkeltoo Mts. (Satyrus).

K. praestans Avinov et Sweadner, 1951. West Tian-Shan: Sandalashsky Mts.; Inner Tian-Shan: Suusamyrtoo Mts.

K. latifasciata (Grum-Grshimailo, 1902). North Tian-Shan: Terskey Ala-Too, Kungey Ala-Too Mts.; Central Tian-Shan: all ridges; Inner Tian-Shan: Naryntoo, Songkeltoo Mts.

K. tancrei (Grum-Grshimailo, 1893). Inner Tian-Shan: At-Bashi Mts. 
K. pungeleri (A.Bang-Haas, 1910). Inner Tian-Shan: Borkoldai, Dzhetim-Bel, Ak-Shiyrak Mts.

K. regeli (Alphéraky, 1881). North Tian-Shan: Transili Alatau, Kungey Ala-Too, Terskey Ala-Too Mts.; Central Tian-Shan: Kaindy, Sarydzhaz, Inylchektau Mts.

K. inopinata Korb, 2014. North Tian-Shan: Kirghizsky Mts.

K. abramovi (Erschoff, 1884). North Tian-Shan: Kirghizsky Mts.; West Tian-Shan: all ridges; Inner Tian-Shan: At-Bashi, Dzhumgal-Too, Baidulu, Dzhetim-Bel, Borkoldai Mts.

Oeneis tarpeia (Pallas, 1771). North Tian-Shan: Transili Alatau, Kungey Ala-Too, Ketmen Mts.; Inner Tian-Shan: Kokshaaltoo Mts.

O. hora Grum-Grshimailo, 1888. North Tian-Shan: all ridges; West Tian-Shan: Fergansky Mts.; Inner Tian-Shan: Baidulu, Naryntoo, Dzhetim-Bel; Central Tian-Shan: all ridges.

Hipparchia autonoe (Esper, [1783]). North Tian-Shan: all ridges; West Tian-Shan: Chatkalsky, Talassky Mts.; Inner Tian-Shan: Suusamyrtoo, Dzhumgal-Too, Kara-Dzhorgo, Naryntoo, Moldo-Too, Baidulu, At-Bashi Mts.; Central Tian-Shan: Kokshaaltoo Mts.

Satyrus ferula (Fabricius, 1793). All ridges.

Minois dryas (Scopoli, 1763). North Tian-Shan: Transili Alatau, Kungey Ala-Too Mts.

Arethusana arethusa ([Denis et Schiffermüller], 1775). North Tian-Shan: Kirghizsky, Kungey Ala-Too, Transili Alatau, Ketmen Mts.; West Tian-Shan: Talassky, Chatkalsky, Fergansky Mts.; Inner Tian-Shan: Suusamyrtoo, Dzhumgaltoo Mts.

Chazara briseis (Linnaeus, 1764). North Tian-Shan: all ridges; West Tian-Shan: all ridges; Inner Tian-Shan: Suusamyrtoo, Dzhumgaltoo, Moldo-Too Mts.

C. enervata (Staudinger, 1881). North Tian-Shan: all ridges; West Tian-Shan: all ridges; Inner Tian-Shan: Naryntoo Mts.

C. kauffmanni (Erschoff, 1874). North Tian-Shan: all ridges; West Tian-Shan: Fergansky Mts.; Inner Tian-Shan: Suusamyrtoo, Dzhumgaltoo, Moldo-Too, At-Bashi Mts.

C. heydenreichi(Lederer, 1853). All ridges.

Pseudochazara esperi Koçak, 1981. North Tian-Shan: Kungey Ala-Too, Terskey Ala-Too, Transili Alatau, Ketmen Mts.

P. turkestana (Grum-Grshimailo, 1893). All ridges.

Hyponephele lycaon (Rottemburg, 1775) (=przhewalskyi Dubatolov, Sergeev et Z[h]danko, 1994). North Tian-Shan: all ridges.

H. Iupina (Costa, [1836]). North Tian-Shan: all ridges; West Tian-Shan: all ridges; Inner Tian-Shan: Suusamyrtoo, Dzhumgaltoo, Moldo-Too, At-Bashi, Borkoldoi Mts.

H. interposita (Erschoff, 1874). North Tian-Shan: Kirghizsky, Kungey Ala-Too, Transili Alatau, Ketmen Mts.; West TianShan: Fergansky Mts.

H. davendra (Moore, 1865). West Tian-Shan: Fergansky Mts.; Inner Tian-Shan: Suusamyrtoo, At-Bashi Mts.

H. dysdora (Lederer, 1870). North Tian-Shan: all ridges; West Tian-Shan: all ridges; Inner Tian-Shan: Suusamyrtoo, Dzhumgaltoo, Moldo-Too, Naryntoo Mts.

H. latistigma (Moore, [1892]). West Tian-Shan: Fergansky, Talassky Mts.; Inner Tian-Shan: Suusamyrtoo,

Dzhumgaltoo, Moldo-Too, Kara-Dzhorgo, Baidulu, Naryntoo, At-Bashi Mts.

H. rueckbeili (Staudinger, 1887). North Tian-Shan: Terskey Ala-Too Mts.; Inner Tian-Shan: Kara-Dzhorgo, Naryntoo, At-Bashi, Baidulu Mts.

H. sheljuzhkoiSamodurov et Tshikolovets, 1996. Inner Tian-Shan: naryntoo Mts.

H. germana (Staudinger, 1887). North Tian-Shan: Kirghizsky, Kungey Ala-Too, Transili Alatau Mts.

H. hilaris (Staudinger, 1886). North Tian-Shan: Kirghizsky Mts.; West Tian-Shan: all ridges; Inner Tian-Shan: Suusamyrtoo, Baidulu, Naryntoo, Moldo-Too, At-Bashi, Kara-Dzhorgo Mts.

H. glasunovi (Grum-Grshimailo, 1893). North Tian-Shan: Kirghizsky Mts.; West Tian-Shan: Talassky, Chatkalsky, Fergansky Mts.; Inner Tian-Shan: Moldo-Too, Naryntoo Mts.

H. cadusina (Staudinger, 1881). North Tian-Shan: Kungey Ala-Too, Terskey Ala-Too, Transili Alatau Mts.

H. naricina (Staudinger, 1870). North Tian-Shan: Kungey Ala-Too, Terskey Ala-Too, Transili Alatau Mts.; West Tian-

Shan: Chatkalsky, Fergansky Mts.; Inner Tian-Shan: Suusamyrtoo, Dzhumgaltoo, Moldo-Too, Kara-Dzhorgo, Baidulu, Naryntoo Mts.

H. kirghisa (Alphéraky, 1881). North Tian-Shan: all ridges.

H. haberhaueri (Staudinger, 1886). North Tian-Shan: Kirghizsky Mts.; West Tian-Shan: all ridges; Inner Tian-Shan: Suusamyrtoo, Dzhumgaltoo, Naryntoo, Moldo-Too, Atbashi Mts.

H. tristis jasavi Lukhtanov, 1990. North Tian-Shan: Kirghizsky Mts.; West Tian-Shan: Chatkalsky, Syrdaryinsky Karatau Mts.

H. korshunovi Lukhtanov, 1994. West Tian-Shan: Chatkalsky Mts.

H. laeta (Staudinger, 1886). North Tian-Shan: Kirghizsky Mts.; West Tian-Shan: all ridges; Inner Tian-Shan: Suusamyrtoo, Dzhumgaltoo Mts.

H. murzini Dubatolov, 1989. West Tian-Shan: Ugamsky, Kuraminsky, Karzhantau, Pskemsky, Chatkalsky Mts.

H. naubidensis (Erschoff, 1874). North Tian-Shan: all ridges; West Tian-Shan: all ridges; Inner Tian-Shan: all ridges. 


\title{
Riodinidae
}

Polycaena tamerlana Staudinger, 1886. All ridges.

P. timur Staudinger, 1886. North Tian-Shan: Kirghizsky, Kungey Ala-Too, Terskey Ala-Too Mts.; Inner Tian-Shan: Baidulu, Naryntoo, Moldo-Too, Kara-Dzhorgo (Polycaena).

\author{
Lycaenidae
}

Cigaritis epargyros (Eversmann, 1854). West Tian-Shan: Fergansky Mts.

Thecla betulae (Linnaeus, 1758). North Tian-Shan: Transili Alatau, Kungey Ala-Too Mts.

Fixsenia sassanides (Kollar, [1849]). West Tian-Shan: all ridges.

F. acaudata (Staudinger, 1901). North Tian-Shan: all ridges; West Tian-Shan: all ridges.

Neolycaena zhdankoi Churkin, 2006. Inner Tian-Shan: Moldo-Too, Naryntoo Mts.

N. tengstroemi (Erschoff, 1874). West Tian-Shan: all ridges.

N. baitenovi (Zhdanko, [2012]) (=halimodendroni Zhdanko, 2012, syn.n.). West Tian-Shan: Syrdaryinsky Karatau Mts.

N. submontanaZhdanko, 1994. North Tian-Shan: all ridges; Inner Tian-Shan: Suusamyrtoo, Dzhumgaltoo Mts.

N. suusamyra Korb, 2010. Inner Tian-Shan: Dzhumgaltoo Mts.

N. carbonaria (Grum-Grshimaïlo, 1890). North Tian-Shan: Kirghizsky, Transili Alatau Mts.

N. baidula Zhdanko, 2000. West Tian-Shan: Talassky Mts.; Inner Tian-Shan: Baidulu, Songkeltoo, Naryntoo, MoldoToo Mts.

N. sinensis (Alphéraky, 1881). North Tian-Shan: all ridges; West Tian-Shan: Chatkalsky, Fergansky Mts.; Inner TianShan: Suusamyrtoo, Dzhumgaltoo, Moldo-Too, Kara-Dzhorgo, Baidulu, Naryntoo Mts. Mts.

N. olga Lukhtanov, 1999. North Tian-Shan: Kirghyzsky Mts.; Inner Tian-Shan: Suusamyrtoo, Moldo-Too, Songkel-Too

N. churkini Zhdanko, 2001. West Tian-Shan: Chatkalsky Mts.

Callophrys rubi (Linnaeus, 1758). North Tian-Shan: all ridges; West Tian-Shan: Fergansky Mts.

C. suaveola (Staudinger, 1881). North Tian-Shan: Ketmen, Kungey Ala-Too, Transili Alatau Mts.; West Tian-Shan: Chatkalsky, Fergansky Mts.

C. titanus Zhdanko, 1998. North Tian-Shan: Kirghizsky Mts.

Ahlbergia arquata Johnson, 1992. Only known by type specimens from Andijan vicinities (West Tian-Shan) in Uzbekistan; no other specimens are known.

Tomares fedtschenkoi (Erschoff, 1874). North Tian-Shan: Kirghizsky, Transili Alatau, Kungey Ala-Too; West Tian-Shan:

Chatkalsky, Ugamsky, Karzhantau Mts.

T. callimachus (Eversmann, 1848). North Tian-Shan: Kirghizsky, Transili Alatau Mts.; West Tian-Shan: Karzhantau, Nuratau, Chatkalsky, Kuraminsky Mts.

Lycaena helle ([Denis et Schiffermüller], 1775). North Tian-Shan: Ketmen Mts.

L. phlaeas (Linnaeus, 1761). All ridges.

L. thersamon (Esper, [1784]). North Tian-Shan: all ridges; West Tian-Shan: all ridges; Inner Tian-Shan: Suusamyrtoo, Dzhumgaltoo, Baidulu, Naryntoo, Moldo-Too Mts.

L. solskyi (Erschoff, 1874). North Tian-Shan: Kirghizsky, Transili Alatau, Kungey Ala-Too, Terskey Ala-Too Mts.; West Tian-Shan: all ridges; Inner Tian-Shan: Dzhumgaltoo, Moldo-Too, Naryntoo, Baidulu, At-Bashi, Borkoldoi Mts.; Central TianShan: Kokshaaltoo Mts.

L. dispar ([Haworth], 1802). North Tian-Shan: all ridges; West Tian-Shan: Chatkalsky, Fergansky Mts.

L. alciphron (Rottemburg, 1775). North Tian-Shan: Kirghizsky, Ketmen, Kungey Ala-Too, Terskey Ala-Too Mts.; Inner Tian-Shan: Naryntoo Mts.

L. splendens (Staudinger, 1881). North Tian-Shan: all ridges; Inner Tian-Shan: all ridges; Central Tian-Shan: all ridges.

L. virgaureae (Linnaeus, 1758). North Tian-Shan: Kirghizsky, Kungey Ala-Too, Terskey Ala-Too Mts.

L. margelanica (Staudinger, 1881). North Tian-Shan: all ridges; West Tian-Shan: all ridges; Inner Tian-Shan: Suusamyrtoo, Dzhumgaltoo, Baidulu, Naryntoo, Moldo-Too, Dzhetim-Bel, At-Bashi Mts.

Athamanthia alexandra (Püngeler, 1901). North Tian-Shan: Kirghizsky, Transili Alatau Mts.; West Tian-Shan: Chatkalsky Mts.; Inner Tian-Shan: Baidulu Mts.

Athamanthia sergetitovi Korb, 2012 (=simurg [Zhdanko, 2012], syn.n.). West Tian-Shan: Syrdaryinsky Karatau Mts.

Note. The name sergetitovi Korb, 2012 published 2 weeks earlier than simurg: publication date of Eversmannia, $\mathrm{N}$ 28-29 is 14.05.2012, publication date of Selevinia, year 2011 is [31.05.2012] (Art. 21.3.1 of ICZN) (it was approved to print 28.04.2012 (see p. 292 of Selevinia, 2011), printed and bounded clearly in May, 2012 (pers. comm. Selevinia's editor A.F.Kovshar), and this is only possible to find out, so Art. 21.3.1 of the Code only is a solution to establish the exact publication date of simurg).

A. dilutior (Staudinger, 1881) (=zhdankoi Lukhtanov, 2000, =churkini Zhdanko, 2000). West Tian-Shan: Chatkalsky, Talassky, Fergansky Mts.; Inner Tian-Shan: Suusamyrtoo, Dzhumgaltoo, Moldo-Too Mts.

A. infera (Nekrutenko, 1984). West Tian-Shan: Chatkalsky, Pskemsky, Karzhantau, Uzunkhamatsky Mts.; Inner TianShan: Suusamyrtoo, Dzhumgaltoo, Moldo-Too Mts. 
A. dimorpha (Staudinger, 1881). North Tian-Shan: Kungey Ala-Too, Terskey Ala-Too, Transili Alatau Mts.; Inner TianShan: Dzhumgaltoo, Suusamyrtoo Mts.

A. namanganica Lukhtanov, 2000. West Tian-Shan: Chatkalsky Mts.

A. issykkuliZhdanko, 1990. North Tian-Shan: Terskey Ala-Too Mts.

Lampides boeticus (Linnaeus, 1767). North Tian-Shan: Kirghizsky, Kungey Ala-Too, Transili Alatau Mts.; West TianShan: Fergansky, Ugamsky, Uzunkhamatsky, Karzhantau, Pskemsky Mts.

Cupido minimus (Fuessly, 1775). North Tian-Shan: Ketmen Mts.

C. buddhista (Alphéraky, 1881). North Tian-Shan: all ridges; West Tian-Shan: all ridges; Inner Tian-Shan: Baidulu Mts.

C. osiris (Meigen, 1829). North Tian-Shan: Kungey Ala-Too, Ketmen, Transili Alatau Mts.; Inner Tian-Shan: all ridges; Inner Tian-Shan: Baidulu Mts.

C. prosecusa (Erschoff, 1874). North Tian-Shan: Kirghizsky, Transili Alatau, Kungey Ala-Too, Terskey Ala-Too Mts.

C. argiades (Pallas, 1771). North Tian-Shan: Transili Alatau Mts.

Celastrina argiolus (Linnaeus, 1758). North Tian-Shan: Kirghizsky, Kungey Ala-Too, Ketmen, Transili Alatau Mts.; West Tian-Shan: all ridges; Inner Tian-Shan: Dzhumgaltoo, Baidulu, Dzhetim-Bel Mts.

Pseudophilotes vicrama (Moore, 1865). North Tian-Shan: all ridges; West Tian-Shan: all ridges; Inner Tian-Shan: Suusamyrtoo, Dzhumgaltoo Mts.

Scolitantides orion (Pallas, 1771). North Tian-Shan: all ridges.

Glaucopsyche alexis (Poda, 1761). North Tian-Shan: all ridges; West Tian-Shan: all ridges; Inner Tian-Shan: Baidulu, Kara-Dzhorgo, Suusamyrtoo, Dzhumgaltoo, Moldo-Too Mts.

G. aeruginosa (Staudinger, 1881). North Tian-Shan: Kirghizsky, Transili Alatau, Kungey Ala-Too Mts.; West Tian-Shan: Talassky, Chatkalsky, Syrdaryinsky Karatau Mts.

G. laetifica (Püngeler, 1898). North Tian-Shan: Kirghizsky Mts.

Phengaris alcon ([Denis et Schiffermüller], [1775]). North Tian-Shan: all ridges; West Tian-Shan: Talassky, Chatkalsky, Sandalashsky Mts.; Inner Tian-Shan: Baidulu, Kara-Dzhorgo, Suusamyrtoo, Dzhumgaltoo, Moldo-Too Mts.

P. teleius (Bergsträsser, 1779). Inner Tian-Shan: Dzhumgaltoo Mts. This species only known from Sary-Kayky mountain massif in Dzhumgaltoo Mts. by one female specimen collected by me in 22-26.06.2009.

P. arion (Linnaeus, 1758). North Tian-Shan: all ridges.

P. cyanecula (Eversmann, 1848). North Tian-Shan: Kirghizsky, Transili Alatau, Kungey Ala-Too Mts.; West Tian-Shan: Talassky, Chatkalsky, Fergansky Mts.; Inner Tian-Shan: Suusamyrtoo, Dzhumgaltoo, Baidulu, Kara-Dzhorgo, Moldo-Too, Naryntoo, At-Bashi Mts.

Turanana tatjana Zhdanko, 1984. North Tian-Shan: Kirghizsky, Transili Alatau, Kungey Ala-Too Mts.

T. panaegides (Staudinger, 1886). North Tian-Shan: Kirghizsky, Kungey Ala-Too Mts.; West Tian-Shan: Talassky, Chatkalsky Mts.; Inner Tian-Shan: Karadzhorgo Mts.

Plebeius argus (Linnaeus, 1758). North Tian-Shan: Kungey Ala-Too, terskey Ala-Too, Ketmen, Transili Alatau Mts.; Inner Tian-Shan: Baidulu, Kara-Dzhorgo Mts.

P. dzhizakiZhdanko, 2000. West Tian-Shan: Kuraminsky Mts.

P. idas (Linnaeus, 1761) (=nushibi Zhdanko, 2000). North Tian-Shan: all ridges; West Tian-Shan: all ridges; Inner TianShan: all ridges.

P. argiva (Staudinger, 1886) (=noah Herz, 1900, =churkini Zhdanko, 2001, =zhdankoi Churkin, 2002, =mellarius Churkin et Zhdanko, 2008, syn.n., =aleremiticus Churkin et Pletnev, 2012). North Tian-Shan: Transili Alatau Mts.; West Tian-Shan: Chatkalsky, Fergansky Mts.; Inner Tian-Shan: Moldo-Too, Naryntoo Mts.

P. christophi (Staudinger, 1874) (=bactriana Grum-Grshimaïlo, 1890). North Tian-Shan: Transili Alatau, Kungey AlaToo Mts.; West Tian-Shan: Fergansky Mts.

P. samudra (Moore, [1875]) (=rogneda Grum-Grshimaillo, 1890, =arpa Churkin et Pletnev, 2012). West Tian-Shan: Fergansky Mts.

P. maracandica (Erschoff, 1874). North Tian-Shan: Kirghizsky, Transili Alatau, Kungey Ala-Too, Terskey Ala-Too Mts.; West Tian-Shan: Fergansky Mts.; Inner Tian-Shan: Moldo-Too Mts.

P. agnata (Staudinger, 1886). North Tian-Shan: Transili Alatau Mts.; West Tian-Shan: Fergansky Mts.; Inner Tian-Shan: At-Bashi Mts.

Afarsia sieversii (Christoph, 1873). West Tian-Shan: Dzhetim-Bel, Nura, Baidulu, Naryntoo, Dzhumgaltoo, Suusamyrtoo Mts.; West Tian-Shan: Chatkalsky, Ugamsky, Pskemsky, Karzhantau Mts.

A. kungeyana (Korb, 2011). North Tian-Shan: Kungey Ala-Too Mts.

A. rutilans (Staudinger, 1886) (=antoninae Lukhtanov, 1999). West Tian-Shan: Talassky, Chatkalsky Mts.

Rueckbeilia fergana (Staudinger, 1881). North Tian-Shan: all ridges; West Tian-Shan: all ridges; Inner Tian-Shan: Suusamyrtoo, Dzhumgaltoo, Baidulu, Kara-Dzhorgo Mts.

R. eversmanni (Lang, 1884). West Tian-Shan: all ridges; Inner Tian-Shan: Dzhumgaltoo Mts.

Agriades pheretiades (Eversmann, 1843). All ridges.

Glabroculus cyane (Eversmann, 1837). North Tian-Shan: all ridges; Inner Tian-Shan: all ridges; Central Tian-Shan: all ridges.

G. elvira (Eversmann, 1854). North Tian-Shan: Transili Alatau, Kungey Ala-Too Mts.; West Tian-Shan: Fergansky Mts.; Inner Tian-Shan: Moldo-Too Mts. 
Aricia agestis ([Denis et Schiffermüller], 1775). North Tian-Shan: all ridges; West Tian-Shan: all ridges; Inner TianShan: Baidulu, Moldo-Too, At-Bashi Mts.

A. artaxerxes (Fabricius, 1793). North Tian-Shan: all ridges; West Tian-Shan: Talassky, Chatkalsky, Karzhantau, Uzunkhamatsky Mts.; Inner Tian-Shan: Moldo-Too, Baidulu, Naryntoo Mts.; Central Tian-Shan: Sary-Dzhaz, Inylchektau Mts.

A. chinensis (Murray, 1874). North Tian-Shan: Transili Alatau, Kirghizsky Mts.

Alpherakya sartus (Alphéraky, 1881). All ridges.

Plebejidea usbekus (Forster, 1939). North Tian-Shan: Kirghizsky Mts.; West Tian-Shan: Talassky, Chatkalsky, Pskemsky, Ugamsky, Karzhantau, Fergansky Mts.

Eumedonia eumedon (Esper, [1780]). All ridges.

E. persephatta (Alphéraky, 1881). All ridges.

Rimisia miris (Staudinger, 1881). North Tian-Shan: Kirghizsky, Transili Alatau Mts.; West Tian-Shan: Chatkalsky, Ugamsky, Pskemsky, Fergansky Mts.

Cyaniris semiargus (Rottemburg, 1775). North Tian-Shan: all ridges; West Tian-Shan: all ridges; Inner Tian-Shan: Baidulu, Kara-Dzhorgo, At-Bashi Mts.

Polyommatus icarus (Rottemburg, 1775). All ridges.

P. icadius (Grum-Grshimaïlo, 1890). North Tian-Shan: all ridges; West Tian-Shan: all ridges; Inner Tian-Shan: Suusamyrtoo, Dzhumgaltoo, Mildo-Too Mts.

P. eros (Ochsenheimer, [1808]). North Tian-Shan: Ketmen, Kungey Ala-Too, Transili Alatau, Terskey Ala-Too Mts.; Inner Tian-Shan: Dzhetim-Bel, Borkoldoi Mts.; Central Tian-Shan: all ridges.

P. venus (Staudinger, 1886). North Tian-Shan: Kirghizsky, Kungey Ala-Too Mts.; West Tian-Shan: all ridges; Inner TianShan: Dzhumgaltoo, Moldo-Too, Baidulu, Dzhetim-Bel Mts.

P. thersites (Cantener, 1834). North Tian-Shan: all ridges; West Tian-Shan: Chatkalsky Mts.; Inner Tian-Shan: baidulu, Dzhetim-Bel, Naryntoo, Dzhumgaltoo Mts.

P. magnificus (Grum-Grshimailo, 1885). West Tian-Shan: Fergansky Mts.

P. ripartii (Freyer, 1830). North Tian-Shan: all ridges; West Tian-Shan: all ridges; Inner Tian-Shan: Dzhumgal-Too, Moldo-Too, Naryntoo, Baidulu, Dzhetim-Bel Mts.

P. damon ([Denis et Schiffermüller], 1775). North Tian-Shan: all ridges; Inner Tian-Shan: Baidulu, Kara-Dzhorgo, Dzhumgaltoo Mts.

P. iphigenides (Staudinger, 1886). North Tian-Shan: Kirghizsky Mts.; West Tian-Shan: all ridges; Inner Tian-Shan: all ridges; Central Tian-Shan: all ridges.

P. juldusus (Staudinger, 1886). North Tian-Shan: Ketmen Mts.

P. kuronjerus Korb, 2011. Inner Tian-Shan: Dzhumgaltoo Mts.

P. karatavicus Lukhtanov, 1990. West Tian-Shan: Syrdaryinsky Karatau Mts.

P. rueckbeili (Forster, 1960). North Tian-Shan: Kurghizsky, Terskey Ala-Too Mts.; Inner Tian-Shan: Baidulu Mts.

P. actinides (Staudinger, 1886). North Tian-Shan: Kirghizsky, Kungey Ala-Too, Transili Alatau Mts.

P. praeactinides (Forster, 1960). West Tian-Shan: Talassky, Chatkalsky, Karzhantau, Pskemsky Mts.

P. phyllides (Staudinger, 1886). North Tian-Shan: Kirghizsky Mts.; West Tian-Shan: all ridges.

P. amandus (Schneider, 1792). North Tian-Shan: all ridges; West Tian-Shan: all ridges; Inner Tian-Shan: Moldo-Too, Naryntoo, Suusamyrtoo, Dzhumgaltoo Mts.

\section{Acknowledgments}

I am greatly indebted to curators of lepidopterological collections used for this study: Prof Dr A.Y. Sinev and Dr A.L. Lvovsky (Zoological Institute, St.-Petersburg, Russia), Dr A.V. Sviridov (Zoological Museum, Moscow State University, Moscow, Russia), Dr W. Mey (Museum für Naturkunde, Berlin, Germany), Dr L. Kaila and Dr J. Kullberg (Finnish Natural History Museum, Helsinki, Finnland), Dr A.G. Tatarinov and O.I. Kulakova (Zoological Museum, Institute of Biology, Komi Science Centre, Syktyvkar, Russia). I am very thankful to D.A. Pozhogin (Nizhny Novgorod, Russia), A.A.Shaposhnikov (Podolsk, Russia), tY.B. Kosarev (Nizhny Novgorod, Russia), L.V. Kaabak (Moscow, Russia) and V.V. Titov (Zheleznodorozhny, Russia) to granting access for materials stored in their private collections.

\section{References}

Alphéraky, S. (1881). Lépidoptères du district de Kouldjà et des montagnes environnantes. Horae Societatis Entomologicae Rossicae, 16, 334-435.

Gvozdetsky, N.A. \& Mikhailov, N.I. (1978). Physical geography of USSR. Asiatic part. Edition 3, reformatted and updated. Moscow: Mysl (in Russian).

Korb, S.K. (2000). A review of the butterfly fauna (Lepidoptera, Rhopalocera) of North Tian-Shan. Zoologichesky Zhurnal, 79, 824-832 (in Russian). 
Korb, S.K. (2011). A distributive list, biotope preferences and flight periods of butterflies of North Tian Shan (Lepidoptera, Diurna). Atalanta, 42, 149-189.

Korb, S.K. (2012). Butterflies (Lepidoptera: Papilionoformes) of North Tian-Shan. Part 1. Hesperiidae, Papilionidae, Pieridae, Libytheidae, Satyridae. Eversmannia, Suppl. 3 (in Russian).

Korb, S.K. (2013). Butterflies (Lepidoptera: Papilionoformes) of North Tian-Shan. Part 2. Nymphalidae, Riodinidae, Lycaenidae. Eversmannia, Suppl 4 (in Russian).

Korb, S.K. (2015). Butterflies of Inner Tian-Shan (Lepidoptera: Papilionoformes). Eversmannia, Suppl. 6 (in Russian).

Korb, S.K. (2017). Butterflies of Central Tian-Shan (Lepidoptera: Papilionoformes). Eversmannia, 39, 24-49 (in Russian).

Staudinger, O. (1886). Centralasiatische Lepidopteren. Stettiner entomologische Zeitung, 47, 193-215, 225-256.

Staudinger, O. (1888). Centralasiatische Lepidopteren. Stettiner entomologische Zeitung, 49, 1-65.

Staudinger, O. (1889). Centralasiatischen Lepidopteren. Stettiner entomologische Zeitung, 50, 16-60.

Tshikolovets, V.V. (2005). The butterflies of Kyrgyzstan. Kiev-Brno: Tshikolovets.

Zhdanko, A.B. (2005). Butterflies (Lepidoptera: Papilionoidea, Hesperioidea) of Kazakhstan. Tethys entomological Research, 11, 85-152.

\section{Citation:}

Korb, S.K. (2017). A distribution list of the butterflies (Lepidoptera, Rhopalocera) of Tian-Shan within the boundaries of the former Soviet Union. Acta Biologica Sibirica, 3 (1), 23-33.

Submitted: 15.12.2016. Accepted: 20.02.2017

cross ref $_{\text {http://dx.doi.org/10.14258/abs.v3i1.2179 }}$

(C) 2017 by the authors. Submitted for possible open access publication under the terms and conditions of the Creative Commons Attribution (CC BY) license (http://creativecommons.org/licenses/by/4.0/). 